Erratum 2: Search for dark matter and unparticles in events with a $Z$ boson and missing transverse momentum in proton-proton collisions at $\sqrt{s}=13 \mathrm{TeV}$

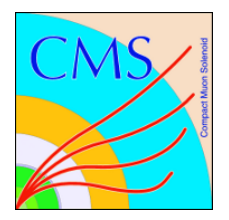

\title{
The CMS collaboration
}

E-mail: cms-publication-committee-chair@cern.ch

ERRATUM TO: JHEP09(2017)106

ARXIV EPRINT: 1701.02042 
In the original paper, figure 10 was incorrect. The correct figure is shown below. Additionally, the unparticle entries in table 3 , as well as figure 4 and 5 were labelled with incorrect values of $\Lambda_{\mathrm{U}}$. The entries correctly correspond to $\Lambda_{\mathrm{U}}=1 \mathrm{TeV}$.

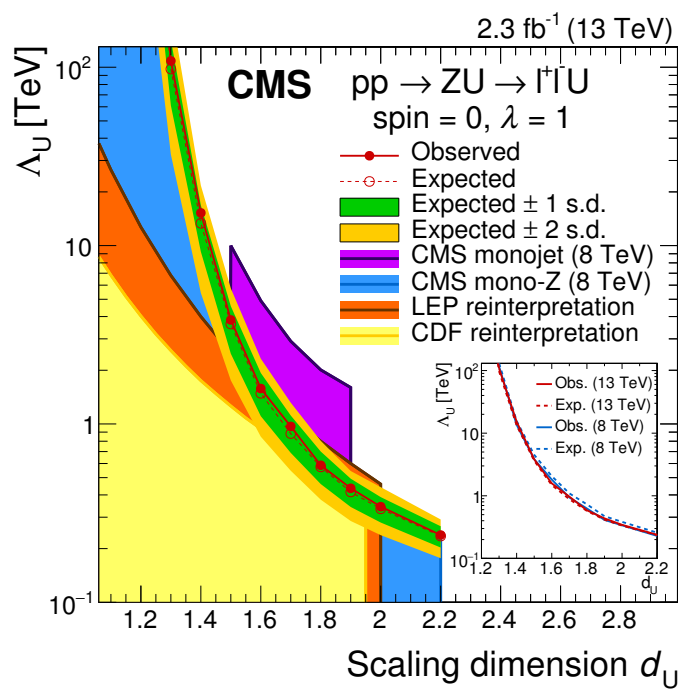

Figure 10. The 95\% CL lower limits on the effective unparticle cutoff scale $\Lambda_{U}$ for a fixed coupling $\lambda=1$. The results from the CMS monojet [1] and mono-Z [2] searches, as well as a reinterpretation of LEP and CDF searches [3] are shown for comparison. The LEP results assume a coupling of unparticles to $\mathrm{Z}$ bosons and photons. The CDF (CMS) monojet result is based on a gluon-unparticle coupling operator (gluon- and quark-unparticle coupling operators). The inset compares the expected and observed limits for the CMS mono-Z analyses at $\sqrt{s}=8$ and $13 \mathrm{TeV}$. Note that the cutoff scales $\Lambda_{U}$ for different operators do not have to be identical. Consequently, the comparison shown here with the results other than the CMS $8 \mathrm{TeV}$ mono- $\mathrm{Z}$ analysis is only qualitative.

Open Access. This article is distributed under the terms of the Creative Commons Attribution License (CC-BY 4.0), which permits any use, distribution and reproduction in any medium, provided the original author(s) and source are credited.

\section{References}

[1] CMS collaboration, Search for dark matter, extra dimensions and unparticles in monojet events in proton-proton collisions at $\sqrt{s}=8$ TeV, Eur. Phys. J. C 75 (2015) 235 [arXiv: 1408.3583] [INSPIRE].

[2] CMS collaboration, Search for dark matter and unparticles produced in association with a $Z$ boson in proton-proton collisions at $\sqrt{s}=8$ TeV, Phys. Rev. D 93 (2016) 052011 [arXiv:1511.09375] [INSPIRE].

[3] S. Kathrein, S. Knapen and M.J. Strassler, Bounds from LEP on unparticle interactions with electroweak bosons, Phys. Rev. D 84 (2011) 015010 [arXiv:1012.3737] [INSPIRE]. 


\section{The CMS collaboration}

\section{Yerevan Physics Institute, Yerevan, Armenia}

A.M. Sirunyan, A. Tumasyan

\section{Institut für Hochenergiephysik, Wien, Austria}

W. Adam, E. Asilar, T. Bergauer, J. Brandstetter, E. Brondolin, M. Dragicevic, J. Erö, M. Flechl, M. Friedl, R. Frühwirth ${ }^{1}$, V.M. Ghete, C. Hartl, N. Hörmann, J. Hrubec, M. Jeitler ${ }^{1}$, A. König, I. Krätschmer, D. Liko, T. Matsushita, I. Mikulec, D. Rabady, N. Rad, B. Rahbaran, H. Rohringer, J. Schieck ${ }^{1}$, J. Strauss, W. Waltenberger, C.-E. Wulz ${ }^{1}$

Institute for Nuclear Problems, Minsk, Belarus

V. Chekhovsky, O. Dvornikov, Y. Dydyshka, I. Emeliantchik, A. Litomin, V. Makarenko, V. Mossolov, R. Stefanovitch, J. Suarez Gonzalez, V. Zykunov

\section{National Centre for Particle and High Energy Physics, Minsk, Belarus}

N. Shumeiko

\section{Universiteit Antwerpen, Antwerpen, Belgium}

S. Alderweireldt, E.A. De Wolf, X. Janssen, J. Lauwers, M. Van De Klundert, H. Van Haevermaet, P. Van Mechelen, N. Van Remortel, A. Van Spilbeeck

\section{Vrije Universiteit Brussel, Brussel, Belgium}

S. Abu Zeid, F. Blekman, J. D'Hondt, N. Daci, I. De Bruyn, K. Deroover, S. Lowette, S. Moortgat, L. Moreels, A. Olbrechts, Q. Python, K. Skovpen, S. Tavernier, W. Van Doninck, P. Van Mulders, I. Van Parijs

\section{Université Libre de Bruxelles, Bruxelles, Belgium}

H. Brun, B. Clerbaux, G. De Lentdecker, H. Delannoy, G. Fasanella, L. Favart, R. Goldouzian, A. Grebenyuk, G. Karapostoli, T. Lenzi, A. Léonard, J. Luetic, T. Maerschalk, A. Marinov, A. Randle-conde, T. Seva, C. Vander Velde, P. Vanlaer, D. Vannerom, R. Yonamine, F. Zenoni, F. Zhang ${ }^{2}$

\section{Ghent University, Ghent, Belgium}

A. Cimmino, T. Cornelis, D. Dobur, A. Fagot, M. Gul, I. Khvastunov, D. Poyraz, S. Salva, R. Schöfbeck, M. Tytgat, W. Van Driessche, E. Yazgan, N. Zaganidis

Université Catholique de Louvain, Louvain-la-Neuve, Belgium

H. Bakhshiansohi, C. Beluffi ${ }^{3}$, O. Bondu, S. Brochet, G. Bruno, A. Caudron, S. De Visscher, C. Delaere, M. Delcourt, B. Francois, A. Giammanco, A. Jafari, M. Komm, G. Krintiras, V. Lemaitre, A. Magitteri, A. Mertens, M. Musich, C. Nuttens, K. Piotrzkowski, L. Quertenmont, M. Selvaggi, M. Vidal Marono, S. Wertz

\section{Université de Mons, Mons, Belgium}

N. Beliy

\section{Centro Brasileiro de Pesquisas Fisicas, Rio de Janeiro, Brazil}

W.L. Aldá Júnior, F.L. Alves, G.A. Alves, L. Brito, C. Hensel, A. Moraes, M.E. Pol, P. Rebello Teles 
Universidade do Estado do Rio de Janeiro, Rio de Janeiro, Brazil

E. Belchior Batista Das Chagas, W. Carvalho, J. Chinellato ${ }^{4}$, A. Custódio, E.M. Da Costa, G.G. Da Silveira ${ }^{5}$, D. De Jesus Damiao, C. De Oliveira Martins, S. Fonseca De Souza, L.M. Huertas Guativa, H. Malbouisson, D. Matos Figueiredo, C. Mora Herrera, L. Mundim, H. Nogima, W.L. Prado Da Silva, A. Santoro, A. Sznajder, E.J. Tonelli Manganote ${ }^{4}$, A. Vilela Pereira

Universidade Estadual Paulista ${ }^{a}$, Universidade Federal do ABC ${ }^{b}$, São Paulo, Brazil

S. Ahuja ${ }^{a}$, C.A. Bernardes ${ }^{a}$, S. Dogra ${ }^{a}$, T.R. Fernandez Perez Tomei $^{a}$, E.M. Gregores ${ }^{b}$, P.G. Mercadante ${ }^{b}$, C.S. Moon ${ }^{a}$, S.F. Novaes ${ }^{a}$, Sandra S. Padula ${ }^{a}$, D. Romero Abad ${ }^{b}$, J.C. Ruiz Vargas ${ }^{a}$

Institute for Nuclear Research and Nuclear Energy, Sofia, Bulgaria

A. Aleksandrov, R. Hadjiiska, P. Iaydjiev, M. Rodozov, S. Stoykova, G. Sultanov, M. Vutova

University of Sofia, Sofia, Bulgaria

A. Dimitrov, I. Glushkov, L. Litov, B. Pavlov, P. Petkov

Beihang University, Beijing, China

W. Fang ${ }^{6}$

Institute of High Energy Physics, Beijing, China

M. Ahmad, J.G. Bian, G.M. Chen, H.S. Chen, M. Chen, Y. Chen ${ }^{7}$, T. Cheng, C.H. Jiang,

D. Leggat, Z. Liu, F. Romeo, M. Ruan, S.M. Shaheen, A. Spiezia, J. Tao, C. Wang,

Z. Wang, H. Zhang, J. Zhao

State Key Laboratory of Nuclear Physics and Technology, Peking University, Beijing, China

Y. Ban, G. Chen, Q. Li, S. Liu, Y. Mao, S.J. Qian, D. Wang, Z. Xu

Universidad de Los Andes, Bogota, Colombia

C. Avila, A. Cabrera, L.F. Chaparro Sierra, C. Florez, J.P. Gomez, C.F. González Hernández, J.D. Ruiz Alvarez, J.C. Sanabria

University of Split, Faculty of Electrical Engineering, Mechanical Engineering and Naval Architecture, Split, Croatia

N. Godinovic, D. Lelas, I. Puljak, P.M. Ribeiro Cipriano, T. Sculac

University of Split, Faculty of Science, Split, Croatia

Z. Antunovic, M. Kovac

Institute Rudjer Boskovic, Zagreb, Croatia

V. Brigljevic, D. Ferencek, K. Kadija, B. Mesic, S. Micanovic, L. Sudic, T. Susa

University of Cyprus, Nicosia, Cyprus

A. Attikis, G. Mavromanolakis, J. Mousa, C. Nicolaou, F. Ptochos, P.A. Razis, H. Rykaczewski, D. Tsiakkouri 
Charles University, Prague, Czech Republic

M. Finger ${ }^{8}$, M. Finger Jr. ${ }^{8}$

Universidad San Francisco de Quito, Quito, Ecuador

E. Carrera Jarrin

Academy of Scientific Research and Technology of the Arab Republic of Egypt, Egyptian Network of High Energy Physics, Cairo, Egypt

A.A. Abdelalim ${ }^{9,10}$, A. Mohamed ${ }^{10}$, A. Mohamed $^{11}$

National Institute of Chemical Physics and Biophysics, Tallinn, Estonia

M. Kadastik, L. Perrini, M. Raidal, A. Tiko, C. Veelken

Department of Physics, University of Helsinki, Helsinki, Finland

P. Eerola, J. Pekkanen, M. Voutilainen

Helsinki Institute of Physics, Helsinki, Finland

J. Härkönen, T. Järvinen, V. Karimäki, R. Kinnunen, T. Lampén, K. Lassila-Perini,

S. Lehti, T. Lindén, P. Luukka, J. Tuominiemi, E. Tuovinen, L. Wendland

Lappeenranta University of Technology, Lappeenranta, Finland

J. Talvitie, T. Tuuva

IRFU, CEA, Université Paris-Saclay, Gif-sur-Yvette, France

M. Besancon, F. Couderc, M. Dejardin, D. Denegri, B. Fabbro, J.L. Faure, C. Favaro, F. Ferri, S. Ganjour, S. Ghosh, A. Givernaud, P. Gras, G. Hamel de Monchenault, P. Jarry, I. Kucher, E. Locci, M. Machet, J. Malcles, J. Rander, A. Rosowsky, M. Titov, A. Zghiche

Laboratoire Leprince-Ringuet, Ecole Polytechnique, IN2P3-CNRS, Palaiseau, France

A. Abdulsalam, I. Antropov, S. Baffioni, F. Beaudette, P. Busson, L. Cadamuro, E. Chapon, C. Charlot, O. Davignon, R. Granier de Cassagnac, M. Jo, S. Lisniak, P. Miné, M. Nguyen, C. Ochando, G. Ortona, P. Paganini, P. Pigard, S. Regnard, R. Salerno, Y. Sirois, T. Strebler, Y. Yilmaz, A. Zabi

Institut Pluridisciplinaire Hubert Curien (IPHC), Université de Strasbourg, CNRS-IN2P3

J.-L. Agram ${ }^{12}$, J. Andrea, A. Aubin, D. Bloch, J.-M. Brom, M. Buttignol, E.C. Chabert, N. Chanon, C. Collard, E. Conte ${ }^{12}$, X. Coubez, J.-C. Fontaine ${ }^{12}$, D. Gelé, U. Goerlach, A.-C. Le Bihan, P. Van Hove

Centre de Calcul de l'Institut National de Physique Nucleaire et de Physique des Particules, CNRS/IN2P3, Villeurbanne, France

S. Gadrat

Université de Lyon, Université Claude Bernard Lyon 1, CNRS-IN2P3, Institut de Physique Nucléaire de Lyon, Villeurbanne, France

S. Beauceron, C. Bernet, G. Boudoul, C.A. Carrillo Montoya, R. Chierici, D. Contardo, B. Courbon, P. Depasse, H. El Mamouni, J. Fan, J. Fay, S. Gascon, M. Gouzevitch, 
G. Grenier, B. Ille, F. Lagarde, I.B. Laktineh, M. Lethuillier, L. Mirabito, A.L. Pequegnot, S. Perries, A. Popov ${ }^{13}$, D. Sabes, V. Sordini, M. Vander Donckt, P. Verdier, S. Viret

\section{Georgian Technical University, Tbilisi, Georgia}

T. Toriashvili ${ }^{14}$

\section{Tbilisi State University, Tbilisi, Georgia}

Z. Tsamalaidze ${ }^{8}$

\section{RWTH Aachen University, I. Physikalisches Institut, Aachen, Germany}

C. Autermann, S. Beranek, L. Feld, M.K. Kiesel, K. Klein, M. Lipinski, M. Preuten, C. Schomakers, J. Schulz, T. Verlage

RWTH Aachen University, III. Physikalisches Institut A, Aachen, Germany A. Albert, M. Brodski, E. Dietz-Laursonn, D. Duchardt, M. Endres, M. Erdmann, S. Erdweg, T. Esch, R. Fischer, A. Güth, M. Hamer, T. Hebbeker, C. Heidemann, K. Hoepfner, S. Knutzen, M. Merschmeyer, A. Meyer, P. Millet, S. Mukherjee, M. Olschewski, K. Padeken, T. Pook, M. Radziej, H. Reithler, M. Rieger, F. Scheuch, L. Sonnenschein, D. Teyssier, S. Thüer

\section{RWTH Aachen University, III. Physikalisches Institut B, Aachen, Germany}

V. Cherepanov, G. Flügge, B. Kargoll, T. Kress, A. Künsken, J. Lingemann, T. Müller, A. Nehrkorn, A. Nowack, C. Pistone, O. Pooth, A. Stahl ${ }^{15}$

\section{Deutsches Elektronen-Synchrotron, Hamburg, Germany}

M. Aldaya Martin, T. Arndt, C. Asawatangtrakuldee, K. Beernaert, O. Behnke, U. Behrens, A.A. Bin Anuar, K. Borras ${ }^{16}$, A. Campbell, P. Connor, C. ContrerasCampana, F. Costanza, C. Diez Pardos, G. Dolinska, G. Eckerlin, D. Eckstein, T. Eichhorn, E. Eren, E. Gallo ${ }^{17}$, J. Garay Garcia, A. Geiser, A. Gizhko, J.M. Grados Luyando, A. Grohsjean, P. Gunnellini, A. Harb, J. Hauk, M. Hempel ${ }^{18}$, H. Jung, A. Kalogeropoulos, O. Karacheban ${ }^{18}$, M. Kasemann, J. Keaveney, C. Kleinwort, I. Korol, D. Krücker, W. Lange, A. Lelek, J. Leonard, K. Lipka, A. Lobanov, W. Lohmann ${ }^{18}$, R. Mankel, I.A. Melzer-Pellmann, A.B. Meyer, G. Mittag, J. Mnich, A. Mussgiller, E. Ntomari, D. Pitzl, R. Placakyte, A. Raspereza, B. Roland, M.Ö. Sahin, P. Saxena, T. Schoerner-Sadenius, C. Seitz, S. Spannagel, N. Stefaniuk, G.P. Van Onsem, R. Walsh, C. Wissing

\section{University of Hamburg, Hamburg, Germany}

V. Blobel, M. Centis Vignali, A.R. Draeger, T. Dreyer, E. Garutti, D. Gonzalez, J. Haller, M. Hoffmann, A. Junkes, R. Klanner, R. Kogler, N. Kovalchuk, T. Lapsien, T. Lenz, I. Marchesini, D. Marconi, M. Meyer, M. Niedziela, D. Nowatschin, F. Pantaleo ${ }^{15}$, T. Peiffer, A. Perieanu, J. Poehlsen, C. Sander, C. Scharf, P. Schleper, A. Schmidt, S. Schumann, J. Schwandt, H. Stadie, G. Steinbrück, F.M. Stober, M. Stöver, H. Tholen, D. Troendle, E. Usai, L. Vanelderen, A. Vanhoefer, B. Vormwald

\section{Institut für Experimentelle Kernphysik, Karlsruhe, Germany}

M. Akbiyik, C. Barth, S. Baur, C. Baus, J. Berger, E. Butz, R. Caspart, T. Chwalek, F. Colombo, W. De Boer, A. Dierlamm, S. Fink, B. Freund, R. Friese, M. Giffels, A. Gilbert, 
P. Goldenzweig, D. Haitz, F. Hartmann ${ }^{15}$, S.M. Heindl, U. Husemann, I. Katkov ${ }^{13}$, S. Kudella, H. Mildner, M.U. Mozer, Th. Müller, M. Plagge, G. Quast, K. Rabbertz, S. Röcker, F. Roscher, M. Schröder, I. Shvetsov, G. Sieber, H.J. Simonis, R. Ulrich, S. Wayand, M. Weber, T. Weiler, S. Williamson, C. Wöhrmann, R. Wolf

Institute of Nuclear and Particle Physics (INPP), NCSR Demokritos, Aghia Paraskevi, Greece

G. Anagnostou, G. Daskalakis, T. Geralis, V.A. Giakoumopoulou, A. Kyriakis, D. Loukas, I. Topsis-Giotis

National and Kapodistrian University of Athens, Athens, Greece

S. Kesisoglou, A. Panagiotou, N. Saoulidou, E. Tziaferi

University of Ioánnina, Ioánnina, Greece

I. Evangelou, G. Flouris, C. Foudas, P. Kokkas, N. Loukas, N. Manthos, I. Papadopoulos, E. Paradas

MTA-ELTE Lendület CMS Particle and Nuclear Physics Group, Eötvös Loránd University, Budapest, Hungary

N. Filipovic

Wigner Research Centre for Physics, Budapest, Hungary

G. Bencze, C. Hajdu, D. Horvath ${ }^{19}$, F. Sikler, V. Veszpremi, G. Vesztergombi ${ }^{20}$, A.J. Zsigmond

Institute of Nuclear Research ATOMKI, Debrecen, Hungary

N. Beni, S. Czellar, J. Karancsi21 ${ }^{21}$ A. Makovec, J. Molnar, Z. Szillasi

Institute of Physics, University of Debrecen

M. Bartók ${ }^{20}$, P. Raics, Z.L. Trocsanyi, B. Ujvari

National Institute of Science Education and Research, Bhubaneswar, India

S. Bahinipati, S. Choudhury ${ }^{22}$, P. Mal, K. Mandal, A. Nayak ${ }^{23}$, D.K. Sahoo, N. Sahoo, S.K. Swain

Panjab University, Chandigarh, India

S. Bansal, S.B. Beri, V. Bhatnagar, R. Chawla, U.Bhawandeep, A.K. Kalsi, A. Kaur, M. Kaur, R. Kumar, P. Kumari, A. Mehta, M. Mittal, J.B. Singh, G. Walia

University of Delhi, Delhi, India

Ashok Kumar, A. Bhardwaj, B.C. Choudhary, R.B. Garg, S. Keshri, S. Malhotra, M. Naimuddin, N. Nishu, K. Ranjan, R. Sharma, V. Sharma

Saha Institute of Nuclear Physics, Kolkata, India

R. Bhattacharya, S. Bhattacharya, K. Chatterjee, S. Dey, S. Dutt, S. Dutta, S. Ghosh, N. Majumdar, A. Modak, K. Mondal, S. Mukhopadhyay, S. Nandan, A. Purohit, A. Roy, D. Roy, S. Roy Chowdhury, S. Sarkar, M. Sharan, S. Thakur

Indian Institute of Technology Madras, Madras, India P.K. Behera 
Bhabha Atomic Research Centre, Mumbai, India

R. Chudasama, D. Dutta, V. Jha, V. Kumar, A.K. Mohanty ${ }^{15}$, P.K. Netrakanti, L.M. Pant, P. Shukla, A. Topkar

Tata Institute of Fundamental Research-A, Mumbai, India

T. Aziz, S. Dugad, G. Kole, B. Mahakud, S. Mitra, G.B. Mohanty, B. Parida, N. Sur, B. Sutar

Tata Institute of Fundamental Research-B, Mumbai, India

S. Banerjee, S. Bhowmik ${ }^{24}$, R.K. Dewanjee, S. Ganguly, M. Guchait, Sa. Jain, S. Kumar, M. Maity ${ }^{24}$, G. Majumder, K. Mazumdar, T. Sarkar ${ }^{24}$, N. Wickramage ${ }^{25}$

Indian Institute of Science Education and Research (IISER), Pune, India

S. Chauhan, S. Dube, V. Hegde, A. Kapoor, K. Kothekar, S. Pandey, A. Rane, S. Sharma

Institute for Research in Fundamental Sciences (IPM), Tehran, Iran

S. Chenarani ${ }^{26}$, E. Eskandari Tadavani, S.M. Etesami ${ }^{26}$, M. Khakzad, M. Mohammadi Najafabadi, M. Naseri, S. Paktinat Mehdiabadi ${ }^{27}$, F. Rezaei Hosseinabadi, B. Safarzadeh ${ }^{28}$, M. Zeinali

University College Dublin, Dublin, Ireland

M. Felcini, M. Grunewald

INFN Sezione di Bari ${ }^{a}$, Università di Bari ${ }^{b}$, Politecnico di Bari ${ }^{c}$, Bari, Italy

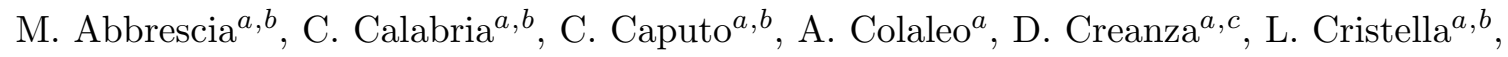
N. De Filippis ${ }^{a, c}$, M. De Palma ${ }^{a, b}$, L. Fiore ${ }^{a}$, G. Iaselli ${ }^{a, c}$, G. Maggi ${ }^{a, c}$, M. Maggi $^{a}$, G. Miniello ${ }^{a, b}$, S. My ${ }^{a, b}$, S. Nuzzo ${ }^{a, b}$, A. Pompili ${ }^{a, b}$, G. Pugliese ${ }^{a, c}$, R. Radogna ${ }^{a, b}$,

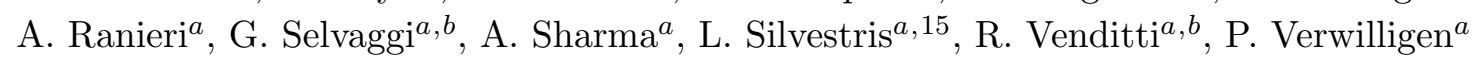

INFN Sezione di Bologna ${ }^{a}$, Università di Bologna ${ }^{b}$, Bologna, Italy

G. Abbiendi ${ }^{a}$, C. Battilana, D. Bonacorsi ${ }^{a, b}$, S. Braibant-Giacomelli ${ }^{a, b}$, L. Brigliadori $^{a, b}$, R. Campanini ${ }^{a, b}$, P. Capiluppi ${ }^{a, b}$, A. Castro ${ }^{a, b}$, F.R. Cavallo $^{a}$, S.S. Chhibra ${ }^{a, b}$, G. Codispoti ${ }^{a, b}$, M. Cuffiani ${ }^{a, b}$, G.M. Dallavalle ${ }^{a}$, F. Fabbri $^{a}$, A. Fanfani ${ }^{a, b}$, D. Fasanella ${ }^{a, b}$, P. Giacomelli ${ }^{a}$, C. Grandi ${ }^{a}$, L. Guiducci ${ }^{a}, b$, S. Marcellini ${ }^{a}$, G. Masetti ${ }^{a}$, A. Montanari ${ }^{a}$,

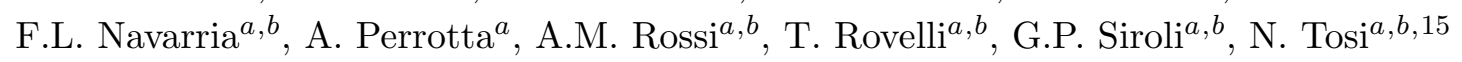

INFN Sezione di Catania ${ }^{a}$, Università di Catania ${ }^{b}$, Catania, Italy

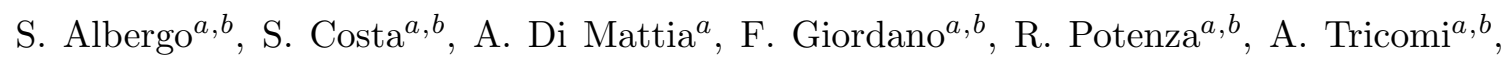
C. Tuve ${ }^{a, b}$

INFN Sezione di Firenze ${ }^{a}$, Università di Firenze ${ }^{b}$, Firenze, Italy

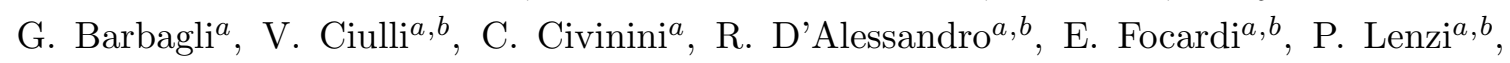
M. Meschini ${ }^{a}$, S. Paoletti ${ }^{a}$, G. Sguazzoni ${ }^{a}$, L. Viliani ${ }^{a, b, 15}$

INFN Laboratori Nazionali di Frascati, Frascati, Italy

L. Benussi, S. Bianco, F. Fabbri, D. Piccolo, F. Primavera ${ }^{15}$

INFN Sezione di Genova ${ }^{a}$, Università di Genova ${ }^{b}$, Genova, Italy

V. Calvelli ${ }^{a, b}$, F. Ferro ${ }^{a}$, M.R. Monge ${ }^{a, b}$, E. Robutti ${ }^{a}$, S. Tosi ${ }^{a, b}$ 
INFN Sezione di Milano-Bicocca ${ }^{a}$, Università di Milano-Bicocca ${ }^{b}$, Milano, Italy

L. Brianza ${ }^{a, b, 15}$, F. Brivio ${ }^{a, b}$, V. Ciriolo, M.E. Dinardo ${ }^{a, b}$, S. Fiorendi ${ }^{a, b, 15}$, S. Gennai ${ }^{a}$,

A. Ghezzi ${ }^{a, b}$, P. Govoni ${ }^{a, b}$, M. Malberti ${ }^{a, b}$, S. Malvezzi ${ }^{a}$, R.A. Manzoni ${ }^{a, b}$, D. Menasce ${ }^{a}$,

L. Moroni ${ }^{a}$, M. Paganoni ${ }^{a, b}$, D. Pedrini ${ }^{a}$, S. Pigazzini ${ }^{a, b}$, S. Ragazzi ${ }^{a, b}$, T. Tabarelli de Fatis $^{a, b}$

INFN Sezione di Napoli ${ }^{a}$, Università di Napoli 'Federico II' ${ }^{b}$, Napoli, Italy, Università della Basilicata ${ }^{c}$, Potenza, Italy, Università G. Marconi ${ }^{d}$, Roma, Italy

S. Buontempo ${ }^{a}$, N. Cavallo ${ }^{a, c}$, G. De Nardo, S. Di Guida ${ }^{a, d, 15}$, M. Esposito ${ }^{a, b}$,

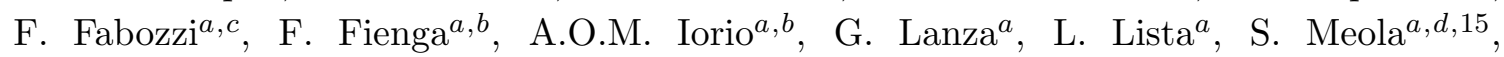
P. Paolucci ${ }^{a, 15}$, C. Sciacca ${ }^{a, b}$, F. Thyssen ${ }^{a}$

INFN Sezione di Padova ${ }^{a}$, Università di Padova ${ }^{b}$, Padova, Italy, Università di Trento ${ }^{c}$, Trento, Italy

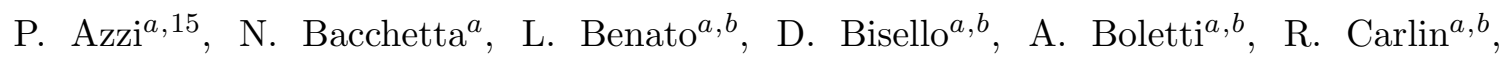
P. Checchia ${ }^{a}$, M. Dall'Osso ${ }^{a, b}$, P. De Castro Manzano ${ }^{a}$, T. Dorigo ${ }^{a}$, U. Gasparini ${ }^{a, b}$, A. Gozzelino ${ }^{a}$, M. Gulmini ${ }^{a, 29}$, S. Lacaprara ${ }^{a}$, M. Margoni ${ }^{a}, b$, G. Maron ${ }^{a, 29}$, A.T. Meneguzzo ${ }^{a, b}$, J. Pazzini ${ }^{a, b}$, N. Pozzobon ${ }^{a, b}$, P. Ronchese ${ }^{a, b}$, F. Simonetto ${ }^{a, b}$, E. Torassa ${ }^{a}$, S. Ventura ${ }^{a}$, M. Zanetti ${ }^{a, b}$, P. Zotto $^{a, b}$, G. Zumerle ${ }^{a, b}$

INFN Sezione di Pavia ${ }^{a}$, Università di Pavia ${ }^{b}$, Pavia, Italy

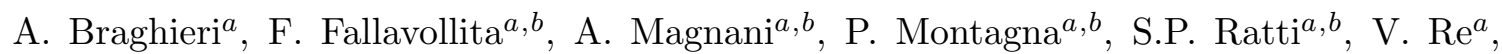
C. Riccardi ${ }^{a}, b$ P. Salvini ${ }^{a}$, I. Vai ${ }^{a, b}$, P. Vitulo ${ }^{a, b}$

INFN Sezione di Perugia ${ }^{a}$, Università di Perugia ${ }^{b}$, Perugia, Italy

L. Alunni Solestizi ${ }^{a, b}$, G.M. Bilei ${ }^{a}$, D. Ciangottini ${ }^{a, b}$, L. Fanò $\grave{o}^{a, b}$, P. Lariccia ${ }^{a}, b$,

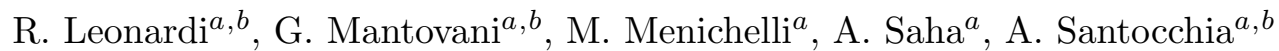

INFN Sezione di Pisa ${ }^{a}$, Università di Pisa ${ }^{b}$, Scuola Normale Superiore di Pisa ${ }^{c}$, Pisa, Italy

K. Androsov ${ }^{a, 30}$, P. Azzurri ${ }^{a, 15}$, G. Bagliesi ${ }^{a}$, J. Bernardini ${ }^{a}$, T. Boccali ${ }^{a}$, R. Castaldi $^{a}$, M.A. Ciocci ${ }^{a, 30}$, R. Dell'Orso ${ }^{a}$, S. Donato ${ }^{a, c}$, G. Fedi, A. Giassi ${ }^{a}$, M.T. Grippo $^{a, 30}$, $^{a}$

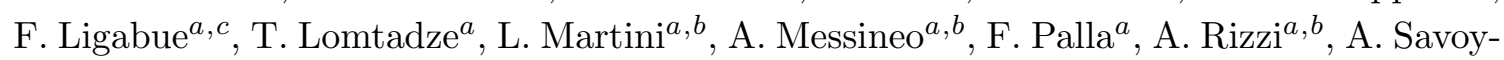

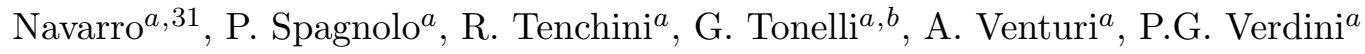

INFN Sezione di Roma ${ }^{a}$, Università di Roma ${ }^{b}$, Roma, Italy

L. Barone ${ }^{a, b}$, F. Cavallari ${ }^{a}$, M. Cipriani ${ }^{a, b}$, D. Del Re Re $^{a, b, 15}$, M. Diemoz ${ }^{a}$, S. Gelli ${ }^{a, b}$, E. Longo ${ }^{a, b}$, F. Margaroli ${ }^{a, b}$, B. Marzocchi ${ }^{a, b}$, P. Meridiani ${ }^{a}$, G. Organtini ${ }^{a, b}$,

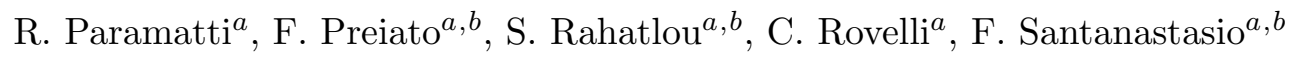

INFN Sezione di Torino ${ }^{a}$, Università di Torino ${ }^{b}$, Torino, Italy, Università del Piemonte Orientale ${ }^{c}$, Novara, Italy

N. Amapane ${ }^{a, b}$, R. Arcidiacono ${ }^{a, c, 15}$, S. Argiro ${ }^{a, b}$, M. Arneodo ${ }^{a, c}$, N. Bartosik ${ }^{a}$, R. Bellan ${ }^{a, b}$, C. Biino ${ }^{a}$, N. Cartiglia ${ }^{a}$, F. Cenna ${ }^{a, b}$, M. Costa ${ }^{a, b}$, R. Covarelli ${ }^{a, b}$, A. Degano ${ }^{a, b}$, N. Demaria ${ }^{a}$, L. Finco ${ }^{a, b}$, B. Kiani ${ }^{a, b}$, C. Mariotti ${ }^{a}$, S. Maselli ${ }^{a}$, 


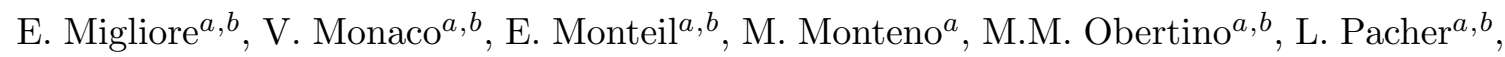
N. Pastrone $^{a}$, M. Pelliccioni ${ }^{a}$, G.L. Pinna Angioni ${ }^{a, b}$, F. Ravera ${ }^{a, b}$, A. Romero ${ }^{a, b}$, M. Ruspa ${ }^{a, c}$, R. Sacchi ${ }^{a, b}$, K. Shchelina ${ }^{a, b}$, V. Sola ${ }^{a}$, A. Solano ${ }^{a, b}$, A. Staiano ${ }^{a}$, P. Traczyk ${ }^{a, b}$

INFN Sezione di Trieste ${ }^{a}$, Università di Trieste ${ }^{b}$, Trieste, Italy

S. Belforte ${ }^{a}$, M. Casarsa ${ }^{a}$, F. Cossutti ${ }^{a}$, G. Della Ricca ${ }^{a}, b$, A. Zanetti ${ }^{a}$

Kyungpook National University, Daegu, Korea

D.H. Kim, G.N. Kim, M.S. Kim, S. Lee, S.W. Lee, Y.D. Oh, S. Sekmen, D.C. Son, Y.C. Yang

Chonbuk National University, Jeonju, Korea

A. Lee

Chonnam National University, Institute for Universe and Elementary Particles, Kwangju, Korea

H. Kim

Hanyang University, Seoul, Korea

J.A. Brochero Cifuentes, T.J. Kim

Korea University, Seoul, Korea

S. Cho, S. Choi, Y. Go, D. Gyun, S. Ha, B. Hong, Y. Jo, Y. Kim, K. Lee, K.S. Lee, S. Lee, J. Lim, S.K. Park, Y. Roh

Seoul National University, Seoul, Korea

J. Almond, J. Kim, H. Lee, S.B. Oh, B.C. Radburn-Smith, S.h. Seo, U.K. Yang, H.D. Yoo, G.B. Yu

University of Seoul, Seoul, Korea

M. Choi, H. Kim, J.H. Kim, J.S.H. Lee, I.C. Park, G. Ryu, M.S. Ryu

Sungkyunkwan University, Suwon, Korea

Y. Choi, J. Goh, C. Hwang, J. Lee, I. Yu

Vilnius University, Vilnius, Lithuania

V. Dudenas, A. Juodagalvis, J. Vaitkus

National Centre for Particle Physics, Universiti Malaya, Kuala Lumpur, Malaysia

I. Ahmed, Z.A. Ibrahim, J.R. Komaragiri, M.A.B. Md Ali ${ }^{32}$, F. Mohamad Idris ${ }^{33}$, W.A.T. Wan Abdullah, M.N. Yusli, Z. Zolkapli

Centro de Investigacion y de Estudios Avanzados del IPN, Mexico City, Mexico

H. Castilla-Valdez, E. De La Cruz-Burelo, I. Heredia-De La Cruz ${ }^{34}$, A. Hernandez-Almada,

R. Lopez-Fernandez, R. Magaña Villalba, J. Mejia Guisao, A. Sanchez-Hernandez

Universidad Iberoamericana, Mexico City, Mexico

S. Carrillo Moreno, C. Oropeza Barrera, F. Vazquez Valencia 
Benemerita Universidad Autonoma de Puebla, Puebla, Mexico

S. Carpinteyro, I. Pedraza, H.A. Salazar Ibarguen, C. Uribe Estrada

Universidad Autónoma de San Luis Potosí, San Luis Potosí, Mexico

A. Morelos Pineda

University of Auckland, Auckland, New Zealand

D. Krofcheck

University of Canterbury, Christchurch, New Zealand

P.H. Butler

National Centre for Physics, Quaid-I-Azam University, Islamabad, Pakistan

A. Ahmad, M. Ahmad, Q. Hassan, H.R. Hoorani, W.A. Khan, A. Saddique, M.A. Shah,

M. Shoaib, M. Waqas

National Centre for Nuclear Research, Swierk, Poland

H. Bialkowska, M. Bluj, B. Boimska, T. Frueboes, M. Górski, M. Kazana, K. Nawrocki,

K. Romanowska-Rybinska, M. Szleper, P. Zalewski

Institute of Experimental Physics, Faculty of Physics, University of Warsaw, Warsaw, Poland

K. Bunkowski, A. Byszuk ${ }^{35}$, K. Doroba, A. Kalinowski, M. Konecki, J. Krolikowski, M. Misiura, M. Olszewski, M. Walczak

Laboratório de Instrumentação e Física Experimental de Partículas, Lisboa, Portugal

P. Bargassa, C. Beirão Da Cruz E Silva, B. Calpas, A. Di Francesco, P. Faccioli, P.G. Ferreira Parracho, M. Gallinaro, J. Hollar, N. Leonardo, L. Lloret Iglesias, M.V. Nemallapudi, J. Rodrigues Antunes, J. Seixas, O. Toldaiev, D. Vadruccio, J. Varela, P. Vischia

Joint Institute for Nuclear Research, Dubna, Russia

S. Afanasiev, V. Alexakhin, P. Bunin, M. Gavrilenko, I. Golutvin, I. Gorbunov, V. Karjavin, A. Lanev, A. Malakhov, V. Matveev ${ }^{36,37}$, V. Palichik, V. Perelygin, M. Savina, S. Shmatov, N. Skatchkov, V. Smirnov, N. Voytishin, A. Zarubin

Petersburg Nuclear Physics Institute, Gatchina (St. Petersburg), Russia

L. Chtchipounov, V. Golovtsov, Y. Ivanov, V. Kim ${ }^{38}$, E. Kuznetsova ${ }^{39}$, V. Murzin, V. Oreshkin, V. Sulimov, A. Vorobyev

Institute for Nuclear Research, Moscow, Russia

Yu. Andreev, A. Dermenev, S. Gninenko, N. Golubev, A. Karneyeu, M. Kirsanov, N. Krasnikov, A. Pashenkov, D. Tlisov, A. Toropin

Institute for Theoretical and Experimental Physics, Moscow, Russia

V. Epshteyn, V. Gavrilov, N. Lychkovskaya, V. Popov, I. Pozdnyakov, G. Safronov, A. Spiridonov, M. Toms, E. Vlasov, A. Zhokin

Moscow Institute of Physics and Technology, Moscow, Russia

A. Bylinkin ${ }^{37}$ 
National Research Nuclear University 'Moscow Engineering Physics Institute' (MEPhI), Moscow, Russia

M. Chadeeva ${ }^{40}$, O. Markin, V. Rusinov

P.N. Lebedev Physical Institute, Moscow, Russia

V. Andreev, M. Azarkin ${ }^{37}$, I. Dremin ${ }^{37}$, M. Kirakosyan, A. Leonidov ${ }^{37}$, A. Terkulov

Skobeltsyn Institute of Nuclear Physics, Lomonosov Moscow State University, Moscow, Russia

A. Baskakov, A. Belyaev, E. Boos, M. Dubinin ${ }^{41}$, L. Dudko, A. Ershov, A. Gribushin, V. Klyukhin, O. Kodolova, I. Lokhtin, I. Miagkov, S. Obraztsov, S. Petrushanko, V. Savrin, A. Snigirev

Novosibirsk State University (NSU), Novosibirsk, Russia

V. Blinov ${ }^{42}$, Y.Skovpen $^{42}$, D. Shtol ${ }^{42}$

State Research Center of Russian Federation, Institute for High Energy Physics, Protvino, Russia

I. Azhgirey, I. Bayshev, S. Bitioukov, D. Elumakhov, V. Kachanov, A. Kalinin, D. Konstantinov, V. Krychkine, V. Petrov, R. Ryutin, A. Sobol, S. Troshin, N. Tyurin, A. Uzunian,

A. Volkov

University of Belgrade, Faculty of Physics and Vinca Institute of Nuclear Sciences, Belgrade, Serbia

P. Adzic ${ }^{43}$, P. Cirkovic, D. Devetak, M. Dordevic, J. Milosevic, V. Rekovic

Centro de Investigaciones Energéticas Medioambientales y Tecnológicas (CIEMAT), Madrid, Spain

J. Alcaraz Maestre, M. Barrio Luna, E. Calvo, M. Cerrada, M. Chamizo Llatas, N. Colino, B. De La Cruz, A. Delgado Peris, A. Escalante Del Valle, C. Fernandez Bedoya, J.P. Fernández Ramos, J. Flix, M.C. Fouz, P. Garcia-Abia, O. Gonzalez Lopez, S. Goy Lopez, J.M. Hernandez, M.I. Josa, E. Navarro De Martino, A. Pérez-Calero Yzquierdo, J. Puerta Pelayo, A. Quintario Olmeda, I. Redondo, L. Romero, M.S. Soares

Universidad Autónoma de Madrid, Madrid, Spain

J.F. de Trocóniz, M. Missiroli, D. Moran

Universidad de Oviedo, Oviedo, Spain

J. Cuevas, J. Fernandez Menendez, I. Gonzalez Caballero, J.R. González Fernández, E. Palencia Cortezon, S. Sanchez Cruz, I. Suárez Andrés, J.M. Vizan Garcia

Instituto de Física de Cantabria (IFCA), CSIC-Universidad de Cantabria, Santander, Spain

I.J. Cabrillo, A. Calderon, J.R. Castiñeiras De Saa, E. Curras, M. Fernandez, J. GarciaFerrero, G. Gomez, A. Lopez Virto, J. Marco, C. Martinez Rivero, F. Matorras, J. Piedra Gomez, T. Rodrigo, A. Ruiz-Jimeno, L. Scodellaro, N. Trevisani, I. Vila, R. Vilar Cortabitarte 


\section{CERN, European Organization for Nuclear Research, Geneva, Switzerland}

D. Abbaneo, E. Auffray, G. Auzinger, M. Bachtis, P. Baillon, A.H. Ball, D. Barney, P. Bloch, A. Bocci, A. Bonato, C. Botta, T. Camporesi, R. Castello, M. Cepeda, G. Cerminara, Y. Chen, D. d'Enterria, A. Dabrowski, V. Daponte, A. David, M. De Gruttola, A. De Roeck, E. Di Marco ${ }^{44}$, M. Dobson, B. Dorney, T. du Pree, D. Duggan, M. Dünser, N. Dupont, A. Elliott-Peisert, P. Everaerts, S. Fartoukh, G. Franzoni, J. Fulcher, W. Funk, D. Gigi, K. Gill, M. Girone, F. Glege, D. Gulhan, S. Gundacker, M. Guthoff, J. Hammer, P. Harris, J. Hegeman, V. Innocente, P. Janot, J. Kieseler, H. Kirschenmann, V. Knünz, A. Kornmayer ${ }^{15}$, M.J. Kortelainen, K. Kousouris, M. Krammer ${ }^{1}$, C. Lange, P. Lecoq, C. Lourenço, M.T. Lucchini, L. Malgeri, M. Mannelli, A. Martelli, F. Meijers, J.A. Merlin, S. Mersi, E. Meschi, P. Milenovic ${ }^{45}$, F. Moortgat, S. Morovic, M. Mulders, H. Neugebauer, S. Orfanelli, L. Orsini, L. Pape, E. Perez, M. Peruzzi, A. Petrilli, G. Petrucciani, A. Pfeiffer, M. Pierini, A. Racz, T. Reis, G. Rolandi ${ }^{46}$, M. Rovere, H. Sakulin, J.B. Sauvan, C. Schäfer, C. Schwick, M. Seidel, A. Sharma, P. Silva, P. Sphicas ${ }^{47}$, J. Steggemann, M. Stoye, Y. Takahashi, M. Tosi, D. Treille, A. Triossi, A. Tsirou, V. Veckalns ${ }^{48}$, G.I. Veres ${ }^{20}$, M. Verweij, N. Wardle, H.K. Wöhri, A. Zagozdzinska ${ }^{35}$, W.D. Zeuner

\section{Paul Scherrer Institut, Villigen, Switzerland}

W. Bertl, K. Deiters, W. Erdmann, R. Horisberger, Q. Ingram, H.C. Kaestli, D. Kotlinski, U. Langenegger, T. Rohe

\section{Institute for Particle Physics, ETH Zurich, Zurich, Switzerland}

F. Bachmair, L. Bäni, L. Bianchini, B. Casal, G. Dissertori, M. Dittmar, M. Donegà, C. Grab, C. Heidegger, D. Hits, J. Hoss, G. Kasieczka, P. Lecomte ${ }^{\dagger}$, W. Lustermann, B. Mangano, M. Marionneau, P. Martinez Ruiz del Arbol, M. Masciovecchio, M.T. Meinhard, D. Meister, F. Micheli, P. Musella, F. Nessi-Tedaldi, F. Pandolfi, J. Pata, F. Pauss, G. Perrin, L. Perrozzi, M. Quittnat, M. Rossini, M. Schönenberger, A. Starodumov ${ }^{49}$, V.R. Tavolaro, K. Theofilatos, R. Wallny

\section{Universität Zürich, Zurich, Switzerland}

T.K. Aarrestad, C. Amsler50, L. Caminada, M.F. Canelli, A. De Cosa, C. Galloni, A. Hinzmann, T. Hreus, B. Kilminster, J. Ngadiuba, D. Pinna, G. Rauco, P. Robmann, D. Salerno, Y. Yang, A. Zucchetta

\section{National Central University, Chung-Li, Taiwan}

V. Candelise, T.H. Doan, Sh. Jain, R. Khurana, M. Konyushikhin, C.M. Kuo, W. Lin, Y.J. Lu, A. Pozdnyakov, S.S. Yu

National Taiwan University (NTU), Taipei, Taiwan

Arun Kumar, P. Chang, Y.H. Chang, Y. Chao, K.F. Chen, P.H. Chen, F. Fiori, W.-S. Hou, Y. Hsiung, Y.F. Liu, R.-S. Lu, M. Miñano Moya, E. Paganis, A. Psallidas, J.f. Tsai

Chulalongkorn University, Faculty of Science, Department of Physics, Bangkok, Thailand

B. Asavapibhop, G. Singh, N. Srimanobhas, N. Suwonjandee 
Cukurova University - Physics Department, Science and Art Faculty

A. Adiguzel, M.N. Bakirci ${ }^{51}$, S. Cerci ${ }^{52}$, S. Damarseckin, Z.S. Demiroglu, C. Dozen, I. Dumanoglu, S. Girgis, G. Gokbulut, Y. Guler, I. $\operatorname{Hos}^{53}$, E.E. Kangal ${ }^{54}$, O. Kara, A. Kayis Topaksu, U. Kiminsu, M. Oglakci, G. Onengut ${ }^{55}$, K. Ozdemir ${ }^{56}$, B. Tali ${ }^{52}$, S. Turkcapar, I.S. Zorbakir, C. Zorbilmez

Middle East Technical University, Physics Department, Ankara, Turkey

B. Bilin, S. Bilmis, B. Isildak ${ }^{57}$, G. Karapinar ${ }^{58}$, M. Yalvac, M. Zeyrek

Bogazici University, Istanbul, Turkey

E. Gülmez, M. Kaya ${ }^{59}$, O. Kaya ${ }^{60}$, E.A. Yetkin ${ }^{61}$, T. Yetkin ${ }^{62}$

Istanbul Technical University, Istanbul, Turkey

A. Cakir, K. Cankocak, S. Sen ${ }^{63}$

Institute for Scintillation Materials of National Academy of Science of Ukraine, Kharkov, Ukraine

B. Grynyov

National Scientific Center, Kharkov Institute of Physics and Technology, Kharkov, Ukraine

L. Levchuk, P. Sorokin

University of Bristol, Bristol, United Kingdom

R. Aggleton, F. Ball, L. Beck, J.J. Brooke, D. Burns, E. Clement, D. Cussans, H. Flacher,

J. Goldstein, M. Grimes, G.P. Heath, H.F. Heath, J. Jacob, L. Kreczko, C. Lucas, D.M. Newbold ${ }^{64}$, S. Paramesvaran, A. Poll, T. Sakuma, S. Seif El Nasr-storey, D. Smith, V.J. Smith

Rutherford Appleton Laboratory, Didcot, United Kingdom

K.W. Bell, A. Belyaev ${ }^{65}$, C. Brew, R.M. Brown, L. Calligaris, D. Cieri, D.J.A. Cockerill, J.A. Coughlan, K. Harder, S. Harper, E. Olaiya, D. Petyt, C.H. Shepherd-Themistocleous, A. Thea, I.R. Tomalin, T. Williams

Imperial College, London, United Kingdom

M. Baber, R. Bainbridge, O. Buchmuller, A. Bundock, D. Burton, S. Casasso, M. Citron,

D. Colling, L. Corpe, P. Dauncey, G. Davies, A. De Wit, M. Della Negra, R. Di Maria,

P. Dunne, A. Elwood, D. Futyan, Y. Haddad, G. Hall, G. Iles, T. James, R. Lane,

C. Laner, R. Lucas ${ }^{64}$, L. Lyons, A.-M. Magnan, S. Malik, L. Mastrolorenzo, J. Nash,

A. Nikitenko ${ }^{49}$, J. Pela, B. Penning, M. Pesaresi, D.M. Raymond, A. Richards, A. Rose,

C. Seez, S. Summers, A. Tapper, K. Uchida, M. Vazquez Acosta ${ }^{66}$, T. Virdee ${ }^{15}$, J. Wright, S.C. Zenz

Brunel University, Uxbridge, United Kingdom

J.E. Cole, P.R. Hobson, A. Khan, P. Kyberd, D. Leslie, I.D. Reid, P. Symonds, L. Teodorescu, M. Turner

Baylor University, Waco, U.S.A.

A. Borzou, K. Call, J. Dittmann, K. Hatakeyama, H. Liu, N. Pastika 
The University of Alabama, Tuscaloosa, U.S.A.

S.I. Cooper, C. Henderson, P. Rumerio, C. West

Boston University, Boston, U.S.A.

D. Arcaro, A. Avetisyan, T. Bose, D. Gastler, D. Rankin, C. Richardson, J. Rohlf, L. Sulak,

D. Zou

\section{Brown University, Providence, U.S.A.}

G. Benelli, D. Cutts, A. Garabedian, J. Hakala, U. Heintz, J.M. Hogan, O. Jesus, K.H.M. Kwok, E. Laird, G. Landsberg, Z. Mao, M. Narain, S. Piperov, S. Sagir, E. Spencer, R. Syarif

University of California, Davis, Davis, U.S.A.

R. Breedon, D. Burns, M. Calderon De La Barca Sanchez, S. Chauhan, M. Chertok, J. Conway, R. Conway, P.T. Cox, R. Erbacher, C. Flores, G. Funk, M. Gardner, W. Ko, R. Lander, C. Mclean, M. Mulhearn, D. Pellett, J. Pilot, S. Shalhout, J. Smith, M. Squires, D. Stolp, M. Tripathi

University of California, Los Angeles, U.S.A.

C. Bravo, R. Cousins, A. Dasgupta, A. Florent, J. Hauser, M. Ignatenko, N. Mccoll, D. Saltzberg, C. Schnaible, V. Valuev, M. Weber

University of California, Riverside, Riverside, U.S.A.

E. Bouvier, K. Burt, R. Clare, J. Ellison, J.W. Gary, S.M.A. Ghiasi Shirazi, G. Hanson, J. Heilman, P. Jandir, E. Kennedy, F. Lacroix, O.R. Long, M. Olmedo Negrete, M.I. Paneva, A. Shrinivas, W. Si, H. Wei, S. Wimpenny, B. R. Yates

University of California, San Diego, La Jolla, U.S.A.

J.G. Branson, G.B. Cerati, S. Cittolin, M. Derdzinski, R. Gerosa, A. Holzner, D. Klein, V. Krutelyov, J. Letts, I. Macneill, D. Olivito, S. Padhi, M. Pieri, M. Sani, V. Sharma, S. Simon, M. Tadel, A. Vartak, S. Wasserbaech ${ }^{67}$, C. Welke, J. Wood, F. Würthwein, A. Yagil, G. Zevi Della Porta

University of California, Santa Barbara - Department of Physics, Santa Barbara, U.S.A.

N. Amin, R. Bhandari, J. Bradmiller-Feld, C. Campagnari, A. Dishaw, V. Dutta, M. Franco Sevilla, C. George, F. Golf, L. Gouskos, J. Gran, R. Heller, J. Incandela, S.D. Mullin, A. Ovcharova, H. Qu, J. Richman, D. Stuart, I. Suarez, J. Yoo

California Institute of Technology, Pasadena, U.S.A.

D. Anderson, J. Bendavid, A. Bornheim, J. Bunn, J. Duarte, J.M. Lawhorn, A. Mott, H.B. Newman, C. Pena, M. Spiropulu, J.R. Vlimant, S. Xie, R.Y. Zhu

Carnegie Mellon University, Pittsburgh, U.S.A.

M.B. Andrews, T. Ferguson, M. Paulini, J. Russ, M. Sun, H. Vogel, I. Vorobiev, M. Weinberg 
University of Colorado Boulder, Boulder, U.S.A.

J.P. Cumalat, W.T. Ford, F. Jensen, A. Johnson, M. Krohn, T. Mulholland, K. Stenson, S.R. Wagner

Cornell University, Ithaca, U.S.A.

J. Alexander, J. Chaves, J. Chu, S. Dittmer, K. Mcdermott, N. Mirman, G. Nicolas Kaufman, J.R. Patterson, A. Rinkevicius, A. Ryd, L. Skinnari, L. Soffi, S.M. Tan, Z. Tao, J. Thom, J. Tucker, P. Wittich, M. Zientek

Fairfield University, Fairfield, U.S.A.

D. Winn

Fermi National Accelerator Laboratory, Batavia, U.S.A.

S. Abdullin, M. Albrow, G. Apollinari, A. Apresyan, S. Banerjee, L.A.T. Bauerdick, A. Beretvas, J. Berryhill, P.C. Bhat, G. Bolla, K. Burkett, J.N. Butler, H.W.K. Cheung, F. Chlebana, S. Cihangir ${ }^{\dagger}$, M. Cremonesi, V.D. Elvira, I. Fisk, J. Freeman, E. Gottschalk, L. Gray, D. Green, S. Grünendahl, O. Gutsche, D. Hare, R.M. Harris, S. Hasegawa, J. Hirschauer, Z. Hu, B. Jayatilaka, S. Jindariani, M. Johnson, U. Joshi, B. Klima, B. Kreis, S. Lammel, J. Linacre, D. Lincoln, R. Lipton, M. Liu, T. Liu, R. Lopes De Sá, J. Lykken, K. Maeshima, N. Magini, J.M. Marraffino, S. Maruyama, D. Mason, P. McBride, P. Merkel, S. Mrenna, S. Nahn, V. O'Dell, K. Pedro, O. Prokofyev, G. Rakness, L. Ristori, E. SextonKennedy, A. Soha, W.J. Spalding, L. Spiegel, S. Stoynev, J. Strait, N. Strobbe, L. Taylor, S. Tkaczyk, N.V. Tran, L. Uplegger, E.W. Vaandering, C. Vernieri, M. Verzocchi, R. Vidal, M. Wang, H.A. Weber, A. Whitbeck, Y. Wu

University of Florida, Gainesville, U.S.A.

D. Acosta, P. Avery, P. Bortignon, D. Bourilkov, A. Brinkerhoff, A. Carnes, M. Carver, D. Curry, S. Das, R.D. Field, I.K. Furic, J. Konigsberg, A. Korytov, J.F. Low, P. Ma, K. Matchev, H. Mei, G. Mitselmakher, D. Rank, L. Shchutska, D. Sperka, L. Thomas, J. Wang, S. Wang, J. Yelton

Florida International University, Miami, U.S.A.

S. Linn, P. Markowitz, G. Martinez, J.L. Rodriguez

Florida State University, Tallahassee, U.S.A.

A. Ackert, T. Adams, A. Askew, S. Bein, S. Hagopian, V. Hagopian, K.F. Johnson, H. Prosper, A. Santra, R. Yohay

\section{Florida Institute of Technology, Melbourne, U.S.A.}

M.M. Baarmand, V. Bhopatkar, S. Colafranceschi, M. Hohlmann, D. Noonan, T. Roy, F. Yumiceva

University of Illinois at Chicago (UIC), Chicago, U.S.A.

M.R. Adams, L. Apanasevich, D. Berry, R.R. Betts, I. Bucinskaite, R. Cavanaugh, O. Evdokimov, L. Gauthier, C.E. Gerber, D.J. Hofman, K. Jung, I.D. Sandoval Gonzalez, N. Varelas, H. Wang, Z. Wu, M. Zakaria, J. Zhang 
The University of Iowa, Iowa City, U.S.A.

B. Bilki ${ }^{68}$, W. Clarida, K. Dilsiz, S. Durgut, R.P. Gandrajula, M. Haytmyradov, V. Khristenko, J.-P. Merlo, H. Mermerkaya ${ }^{69}$, A. Mestvirishvili, A. Moeller, J. Nachtman, H. Ogul, Y. Onel, F. Ozok ${ }^{70}$, A. Penzo, C. Snyder, E. Tiras, J. Wetzel, K. Yi

Johns Hopkins University, Baltimore, U.S.A.

I. Anderson, B. Blumenfeld, A. Cocoros, N. Eminizer, D. Fehling, L. Feng, A.V. Gritsan, P. Maksimovic, C. Martin, M. Osherson, J. Roskes, U. Sarica, M. Swartz, M. Xiao, Y. Xin, C. You

The University of Kansas, Lawrence, U.S.A.

A. Al-bataineh, P. Baringer, A. Bean, S. Boren, J. Bowen, J. Castle, L. Forthomme, R.P. Kenny III, S. Khalil, A. Kropivnitskaya, D. Majumder, W. Mcbrayer, M. Murray, S. Sanders, R. Stringer, J.D. Tapia Takaki, Q. Wang

Kansas State University, Manhattan, U.S.A.

A. Ivanov, K. Kaadze, Y. Maravin, A. Mohammadi, L.K. Saini, N. Skhirtladze, S. Toda

Lawrence Livermore National Laboratory, Livermore, U.S.A.

F. Rebassoo, D. Wright

University of Maryland, College Park, U.S.A.

C. Anelli, A. Baden, O. Baron, A. Belloni, B. Calvert, S.C. Eno, C. Ferraioli, J.A. Gomez, N.J. Hadley, S. Jabeen, R.G. Kellogg, T. Kolberg, J. Kunkle, Y. Lu, A.C. Mignerey, F. Ricci-Tam, Y.H. Shin, A. Skuja, M.B. Tonjes, S.C. Tonwar

Massachusetts Institute of Technology, Cambridge, U.S.A.

D. Abercrombie, B. Allen, A. Apyan, V. Azzolini, R. Barbieri, A. Baty, R. Bi, K. Bierwagen, S. Brandt, W. Busza, I.A. Cali, M. D’Alfonso, Z. Demiragli, L. Di Matteo, G. Gomez Ceballos, M. Goncharov, D. Hsu, Y. Iiyama, G.M. Innocenti, M. Klute, D. Kovalskyi, K. Krajczar, Y.S. Lai, Y.-J. Lee, A. Levin, P.D. Luckey, B. Maier, A.C. Marini, C. Mcginn, C. Mironov, S. Narayanan, X. Niu, C. Paus, C. Roland, G. Roland, J. Salfeld-Nebgen, G.S.F. Stephans, K. Tatar, M. Varma, D. Velicanu, J. Veverka, J. Wang, T.W. Wang, B. Wyslouch, M. Yang

University of Minnesota, Minneapolis, U.S.A.

A.C. Benvenuti, R.M. Chatterjee, A. Evans, P. Hansen, S. Kalafut, S.C. Kao, Y. Kubota, Z. Lesko, J. Mans, S. Nourbakhsh, N. Ruckstuhl, R. Rusack, N. Tambe, J. Turkewitz

University of Mississippi, Oxford, U.S.A.

J.G. Acosta, S. Oliveros

University of Nebraska-Lincoln, Lincoln, U.S.A.

E. Avdeeva, R. Bartek ${ }^{71}$, K. Bloom, D.R. Claes, A. Dominguez ${ }^{71}$, C. Fangmeier, R. Gonzalez Suarez, R. Kamalieddin, I. Kravchenko, A. Malta Rodrigues, F. Meier, J. Monroy, J.E. Siado, G.R. Snow, B. Stieger 
State University of New York at Buffalo, Buffalo, U.S.A.

M. Alyari, J. Dolen, A. Godshalk, C. Harrington, I. Iashvili, J. Kaisen, A. Kharchilava, A. Parker, S. Rappoccio, B. Roozbahani

Northeastern University, Boston, U.S.A.

G. Alverson, E. Barberis, A. Hortiangtham, A. Massironi, D.M. Morse, D. Nash, T. Orimoto, R. Teixeira De Lima, D. Trocino, R.-J. Wang, D. Wood

Northwestern University, Evanston, U.S.A.

S. Bhattacharya, O. Charaf, K.A. Hahn, A. Kumar, N. Mucia, N. Odell, B. Pollack, M.H. Schmitt, K. Sung, M. Trovato, M. Velasco

University of Notre Dame, Notre Dame, U.S.A.

N. Dev, M. Hildreth, K. Hurtado Anampa, C. Jessop, D.J. Karmgard, N. Kellams, K. Lannon, N. Marinelli, F. Meng, C. Mueller, Y. Musienko ${ }^{36}$, M. Planer, A. Reinsvold, R. Ruchti, G. Smith, S. Taroni, M. Wayne, M. Wolf, A. Woodard

The Ohio State University, Columbus, U.S.A.

J. Alimena, L. Antonelli, B. Bylsma, L.S. Durkin, S. Flowers, B. Francis, A. Hart, C. Hill, R. Hughes, W. Ji, B. Liu, W. Luo, D. Puigh, B.L. Winer, H.W. Wulsin

Princeton University, Princeton, U.S.A.

S. Cooperstein, O. Driga, P. Elmer, J. Hardenbrook, P. Hebda, D. Lange, J. Luo, D. Marlow, T. Medvedeva, K. Mei, J. Olsen, C. Palmer, P. Piroué, D. Stickland, A. Svyatkovskiy, C. Tully

University of Puerto Rico, Mayaguez, U.S.A.

S. Malik

Purdue University, West Lafayette, U.S.A.

A. Barker, V.E. Barnes, S. Folgueras, L. Gutay, M.K. Jha, M. Jones, A.W. Jung, A. Khatiwada, D.H. Miller, N. Neumeister, J.F. Schulte, X. Shi, J. Sun, F. Wang, W. Xie Purdue University Calumet, Hammond, U.S.A.

N. Parashar, J. Stupak

Rice University, Houston, U.S.A.

A. Adair, B. Akgun, Z. Chen, K.M. Ecklund, F.J.M. Geurts, M. Guilbaud, W. Li, B. Michlin, M. Northup, B.P. Padley, J. Roberts, J. Rorie, Z. Tu, J. Zabel

University of Rochester, Rochester, U.S.A.

B. Betchart, A. Bodek, P. de Barbaro, R. Demina, Y.t. Duh, T. Ferbel, M. Galanti, A. Garcia-Bellido, J. Han, O. Hindrichs, A. Khukhunaishvili, K.H. Lo, P. Tan, M. Verzetti

Rutgers, The State University of New Jersey, Piscataway, U.S.A.

A. Agapitos, J.P. Chou, Y. Gershtein, T.A. Gómez Espinosa, E. Halkiadakis, M. Heindl, E. Hughes, S. Kaplan, R. Kunnawalkam Elayavalli, S. Kyriacou, A. Lath, K. Nash, H. Saka, S. Salur, S. Schnetzer, D. Sheffield, S. Somalwar, R. Stone, S. Thomas, P. Thomassen, M. Walker 
University of Tennessee, Knoxville, U.S.A.

A.G. Delannoy, M. Foerster, J. Heideman, G. Riley, K. Rose, S. Spanier, K. Thapa

Texas A\&M University, College Station, U.S.A.

O. Bouhali ${ }^{72}$, A. Celik, M. Dalchenko, M. De Mattia, A. Delgado, S. Dildick, R. Eusebi, J. Gilmore, T. Huang, E. Juska, T. Kamon ${ }^{73}$, R. Mueller, Y. Pakhotin, R. Patel, A. Perloff,

L. Perniè, D. Rathjens, A. Safonov, A. Tatarinov, K.A. Ulmer

Texas Tech University, Lubbock, U.S.A.

N. Akchurin, C. Cowden, J. Damgov, F. De Guio, C. Dragoiu, P.R. Dudero, J. Faulkner, E. Gurpinar, S. Kunori, K. Lamichhane, S.W. Lee, T. Libeiro, T. Peltola, S. Undleeb, I. Volobouev, Z. Wang

Vanderbilt University, Nashville, U.S.A.

S. Greene, A. Gurrola, R. Janjam, W. Johns, C. Maguire, A. Melo, H. Ni, P. Sheldon, S. Tuo, J. Velkovska, Q. Xu

University of Virginia, Charlottesville, U.S.A.

M.W. Arenton, P. Barria, B. Cox, J. Goodell, R. Hirosky, A. Ledovskoy, H. Li, C. Neu, T. Sinthuprasith, X. Sun, Y. Wang, E. Wolfe, F. Xia

Wayne State University, Detroit, U.S.A.

C. Clarke, R. Harr, P.E. Karchin, J. Sturdy

University of Wisconsin - Madison, Madison, WI, U.S.A.

D.A. Belknap, J. Buchanan, C. Caillol, S. Dasu, L. Dodd, S. Duric, B. Gomber, M. Grothe, M. Herndon, A. Hervé, P. Klabbers, A. Lanaro, A. Levine, K. Long, R. Loveless, I. Ojalvo, T. Perry, G.A. Pierro, G. Polese, T. Ruggles, A. Savin, N. Smith, W.H. Smith, D. Taylor, N. Woods

$\dagger$ : Deceased

1: Also at Vienna University of Technology, Vienna, Austria

2: Also at State Key Laboratory of Nuclear Physics and Technology, Peking University, Beijing, China

3: Also at Institut Pluridisciplinaire Hubert Curien (IPHC), Université de Strasbourg, CNRS/IN2P3, Strasbourg, France

4: Also at Universidade Estadual de Campinas, Campinas, Brazil

5: Also at Universidade Federal de Pelotas, Pelotas, Brazil

6: Also at Université Libre de Bruxelles, Bruxelles, Belgium

7: Also at Deutsches Elektronen-Synchrotron, Hamburg, Germany

8: Also at Joint Institute for Nuclear Research, Dubna, Russia

9: Also at Helwan University, Cairo, Egypt

10: Now at Zewail City of Science and Technology, Zewail, Egypt

11: Also at Ain Shams University, Cairo, Egypt

12: Also at Université de Haute Alsace, Mulhouse, France

13: Also at Skobeltsyn Institute of Nuclear Physics, Lomonosov Moscow State University, Moscow, Russia

14: Also at Tbilisi State University, Tbilisi, Georgia 
15: Also at CERN, European Organization for Nuclear Research, Geneva, Switzerland

16: Also at RWTH Aachen University, III. Physikalisches Institut A, Aachen, Germany

17: Also at University of Hamburg, Hamburg, Germany

18: Also at Brandenburg University of Technology, Cottbus, Germany

19: Also at Institute of Nuclear Research ATOMKI, Debrecen, Hungary

20: Also at MTA-ELTE Lendület CMS Particle and Nuclear Physics Group, Eötvös Loránd University, Budapest, Hungary

21: Also at Institute of Physics, University of Debrecen, Debrecen, Hungary

22: Also at Indian Institute of Science Education and Research, Bhopal, India

23: Also at Institute of Physics, Bhubaneswar, India

24: Also at University of Visva-Bharati, Santiniketan, India

25: Also at University of Ruhuna, Matara, Sri Lanka

26: Also at Isfahan University of Technology, Isfahan, Iran

27: Also at Yazd University, Yazd, Iran

28: Also at Plasma Physics Research Center, Science and Research Branch, Islamic Azad University, Tehran, Iran

29: Also at Laboratori Nazionali di Legnaro dell'INFN, Legnaro, Italy

30: Also at Università degli Studi di Siena, Siena, Italy

31: Also at Purdue University, West Lafayette, U.S.A.

32: Also at International Islamic University of Malaysia, Kuala Lumpur, Malaysia

33: Also at Malaysian Nuclear Agency, MOSTI, Kajang, Malaysia

34: Also at Consejo Nacional de Ciencia y Tecnología, Mexico city, Mexico

35: Also at Warsaw University of Technology, Institute of Electronic Systems, Warsaw, Poland

36: Also at Institute for Nuclear Research, Moscow, Russia

37: Now at National Research Nuclear University 'Moscow Engineering Physics Institute' (MEPhI), Moscow, Russia

38: Also at St. Petersburg State Polytechnical University, St. Petersburg, Russia

39: Also at University of Florida, Gainesville, U.S.A.

40: Also at P.N. Lebedev Physical Institute, Moscow, Russia

41: Also at California Institute of Technology, Pasadena, U.S.A.

42: Also at Budker Institute of Nuclear Physics, Novosibirsk, Russia

43: Also at Faculty of Physics, University of Belgrade, Belgrade, Serbia

44: Also at INFN Sezione di Roma; Università di Roma, Roma, Italy

45: Also at University of Belgrade, Faculty of Physics and Vinca Institute of Nuclear Sciences, Belgrade, Serbia

46: Also at Scuola Normale e Sezione dell'INFN, Pisa, Italy

47: Also at National and Kapodistrian University of Athens, Athens, Greece

48: Also at Riga Technical University, Riga, Latvia

49: Also at Institute for Theoretical and Experimental Physics, Moscow, Russia

50: Also at Albert Einstein Center for Fundamental Physics, Bern, Switzerland

51: Also at Gaziosmanpasa University, Tokat, Turkey

52: Also at Adiyaman University, Adiyaman, Turkey

53: Also at Istanbul Aydin University, Istanbul, Turkey

54: Also at Mersin University, Mersin, Turkey

55: Also at Cag University, Mersin, Turkey

56: Also at Piri Reis University, Istanbul, Turkey

57: Also at Ozyegin University, Istanbul, Turkey

58: Also at Izmir Institute of Technology, Izmir, Turkey

59: Also at Marmara University, Istanbul, Turkey 
60: Also at Kafkas University, Kars, Turkey

61: Also at Istanbul Bilgi University, Istanbul, Turkey

62: Also at Yildiz Technical University, Istanbul, Turkey

63: Also at Hacettepe University, Ankara, Turkey

64: Also at Rutherford Appleton Laboratory, Didcot, United Kingdom

65: Also at School of Physics and Astronomy, University of Southampton, Southampton, United Kingdom

66: Also at Instituto de Astrofísica de Canarias, La Laguna, Spain

67: Also at Utah Valley University, Orem, U.S.A.

68: Also at Argonne National Laboratory, Argonne, U.S.A.

69: Also at Erzincan University, Erzincan, Turkey

70: Also at Mimar Sinan University, Istanbul, Istanbul, Turkey

71: Now at The Catholic University of America, Washington, U.S.A.

72: Also at Texas A\&M University at Qatar, Doha, Qatar

73: Also at Kyungpook National University, Daegu, Korea 